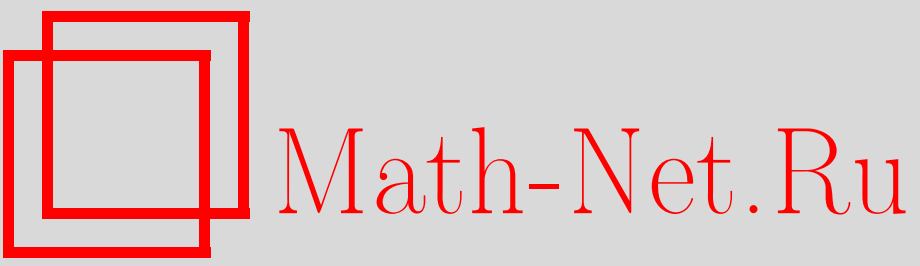

М. А. Степович, Д. В. Туртин, Е. В. Серегина, О корректности математических моделей диффузии, обусловленной остро сфокусированным электронным зондом в однородном полупроводниковом материале, Итоги науки и техн. Сер. Соврем. мат. и ее прил. Темат. обз., 2021, том 193, 122-129

DOI: https://doi.org/10.36535/0233-6723-2021-193-122-129

Использование Общероссийского математического портала Math-Net.Ru подразумевает, что вы прочитали и согласны с пользовательским соглашением

http://www.mathnet.ru/rus/agreement

Параметры загрузки:

IP : 54.172 .240 .79

26 апреля 2023 г., $16: 20: 38$ 


\title{
О КОРРЕКТНОСТИ МАТЕМАТИЧЕСКИХ МОДЕЛЕЙ ДИФФУЗИИ, ОБУСЛОВЛЕННОЙ ОСТРО СФОКУСИРОВАННЫМ ЭЛЕКТРОННЫМ ЗОНДОМ В ОДНОРОДНОМ ПОЛУПРОВОДНИКОВОМ МАТЕРИАЛЕ
}

\author{
(C) 2021 г. М. А. СТЕПОВИЧ, Д. В. ТУРТИН, Е. В. СЕРЕГИНА
}

\begin{abstract}
АннотАция. Проведено сравнительное исследование качественных свойств двумерной и трехмерной математических моделей диффузии частиц (неравновесных неосновных носителей заряда, экситонов), генерируемых остро сфокусированным электронным зондом в однородном полупроводниковом материале. Показано, что рассматриваемые математические модели являются математически корректными и могут быть применены для оценки электрофизических параметров однородных полупроводниковых мишеней по результатам экспериментальных измерений.
\end{abstract}

Ключевъе слова: математическая модель, дифференциальное уравнение, частная производная, задача Коши, электронный зонд, полупроводник, корректность, единственность, непрерывная зависимость от данных, идентификация.

\section{ON THE WELL-POSEDNESS OF MATHEMATICAL MODELS OF DIFFUSION DUE TO A SHARPLY FOCUSED ELECTRON PROBE IN A HOMOGENEOUS SEMICONDUCTOR MATERIAL}

\author{
(c) 2021 M. A. STEPOVICH, D. V. TURTIN, E. V. SEREGINA
}

\begin{abstract}
In this paper, we compare qualitative properties of two- and three-dimensional mathematical models of the diffusion of particles (nonequilibrium minority charge carriers, excitons) generated by a sharply focused electron probe in a homogeneous semiconductor material. We show that the mathematical models considered are well posed and can be used for estimating electrophysical parameters of homogeneous semiconductor targets based on the results of experimental measurements.
\end{abstract}

Keywords and phrases: mathematical model, differential equation, partial derivative, Cauchy problem, electron probe, semiconductor, well-posedness, uniqueness, continuous dependence on data, identification.

AMS Subject Classification: 35G16, 35A02, 78A35

1. Введение. Значительное число различных процессов описывается начальными или краевыми задачами для уравнений с частными производными. При математическом моделировании таких процессов необходимо не только адекватно описать суть изучаемых явлений, но и исследовать проблемы существования решения рассматриваемых задач, доказать единственность решения, обосновать непрерывную зависимость решения от данных задачи (начальных и граничных данных, свободного члена, коэффициентов операторного уравнения). Задачи, удовлетворяющие

Работа выполнена при поддержке Российского фонда фундаментальных исследований и правительства Калужской области (проекты № 19-03-00271, № 18-41-400001). 
вышеуказанным требованиям, называются корректно поставленными. Вопросы существования и единственности решения задачи Коши и краевых задач являются традиционными и имеют богатую историю в теории уравнений в частных производных (см., например, [2, 13]). Исследование таких задач очень актуально, поскольку в реальных задачах начальные данные и параметры задачи имеют погрешность измерения, и, как показывает классический пример Ж. Адамара (см., например, [2]), при незначительных изменениях начальных данных решения задачи могут резко различаться. Однако проблемы корректности подобных математических моделей изучаются довольно редко в связи с большими трудностями логического и технического характера, что так же делает такие исследования весьма актуальными.

В настоящей работе в качестве исходных моделей, подлежащих рассмотрению, использованы двумерная и трехмерная математические модели диффузии частиц (экситонов или неравновесных неосновных носителей заряда (НН3, см. [1,6]), генерированных остро сфокусированным электронным пучком (электронным зондом) в однородном полупроводниковом материале.

В $[9-11,14]$ приведены исходные формулы, количественно описывающие двумерную диффузию частиц, генерированных электронным зондом в полупроводниковом монокристаллическом нитриде галлия для стационарного случая постоянного облучения полупроводника и нестационарного процесса, возникающего после прекращения облучения. Приведены некоторые результаты расчетов с использованием этих формул и сравнение с экспериментом с целью идентификации электрофизических параметров исследуемого полупроводникового материала. Однако математически корректного анализа предложенных двумерных математических моделей в этих работах не проводилось. Результаты такого рассмотрения для двумерной диффузии экситонов, генерированных в монокристаллическом нитриде галлия, изложены в [12,15]. В этих работах рассмотрены узловые вопросы, связанные с единственностью в плоскости и непрерывной зависимостью решения задачи от члена в правой части дифференциальных уравнений стационарной и нестационарной диффузии, описывающего распределение частиц, генерированных электронным зондом. Подробное рассмотрение такой математической модели с доказательством ее корректности изложено B [17].

Использование модели двумерной диффузии можно считать приемлемым для низких энергий электронов зонда, примерно до 6..8 кэВ. При этом толщина приповерхностного слоя мишени, в котором теряется основная часть энергии электронов, составляет не более 0,4 .. 0,5 мкм для легких полупроводниковых мишеней, например, кремния, не более 0,1 мкм для полупроводников со средними атомными номерами, например, для арсенида галлия, и не более 0,03 мкм для тяжелых полупроводников, например, теллурида кадмия (см. [3-5, 16]. В большинстве вышеуказанных работ при моделировании процесса потерь энергии первичными электронами при их взаимодействии с конденсированным веществом использовалась модель, описывающая плотность потерь энергии в мишени $\rho^{*}(M)$ (а, следовательно, и распределение генерированных частиц) в виде двумерного нормального распределения Гаусса. В связи с этим отметим, что лучшее согласие с экспериментом достигается при использовании математической модели, основанной на раздельном описании потерь энергии электронами, испытавшими малоугловое рассеяние и поглощенными мишенью и электронами, испытавшими отражение на большие углы и покинувшие мишень (см. [3-5]). Такой подход для описания плотности потерь энергии электронным зондом в твердом теле (а, значит, и области генерации неравновесных частиц) лучше отвечает реальным потерям энергии пучком первичных электронов в твердом теле и его применение наиболее оправдано для трехмерных моделей диффузии. В то же время и подход, основанный на использовании функции Гаусса, для ряда задач является вполне приемлемым, потому он используется и в настоящей работе. Отметим также, что в настоящее время имеются математические модели, описывающие трехмерную диффузию экситонов, генерированных электронным зондом в полупроводниковой мишени (см. [7,8]), однако математически корректный анализ предлагаемых моделей в этих работах не проводился.

Оценка корректности двумерной и трехмерной математических моделей диффузии частиц, генерированных электронным зондом в полупроводниковой мишени и учитывающих основные 
особенности потерь энергии в конденсированном веществе и составляет предмет рассмотрения в настоящей работе.

2. Математические модели изучаемых процессов диффузии. В общем случае при использовании электронного зонда реализуется трехмерная диффузия неравновесных ННЗ. Соответствующее уравнение стационарной диффузии для ННЗ имеет вид (см. [7, 8]):

$$
a^{2} \operatorname{div}[\operatorname{grad} \Delta p(M)]-\Delta p(M)=-\rho(M)
$$

с граничными условиями

$$
\left.D \frac{d \Delta p(M)}{d z}\right|_{z=0}=v_{s} \Delta p(x, y, 0), \quad \Delta p(\infty, \infty, \infty)=0 .
$$

Функция $\Delta p(M)$ описывает распределение диффундирующего вещества; $M(x, y, z)$ - произвольная точка мишени; $x, y \in(-\infty, \infty), z \in[0, \infty), a=\mathrm{const}$, а коэффициент диффузии $D$ и скорость поверхностной рекомбинации ННЗ $v_{s}$ для однородного полупроводника также постоянные величины. Отметим, что при моделировании процесса диффузии в правой части уравнения (1) должна находиться концентрация генерированных в полупроводнике ННЗ $\rho(M)$, что достигается делением $\rho^{*}(M)$ на энергию образования частицы: электронно-дырочной пары или экситона (см. $[1,6])$.

Будем рассматривать отдельно математические модели, описывающие двумерную и трехмерную диффузию ННЗ для стационарного (количество генерированных и рекомбинировавших частиц в единицу времени постоянно и равно друг другу, что соответствует состоянию квазиравновесия) и нестационарного (электронный пучок выключается, генерации частиц нет, имеется лишь их рекомбинации) процессов. Поскольку двумерная модель диффузии и вопросы ее корректности достаточно подробно рассмотрены ранее (см. ссылки на литературу в разделе «Введение»), за основу изложения полученных результатов сравнительного анализа примем трехмерную модель, параметры которой в декартовой прямоугольной системе координат будут зависеть от трех переменных: $x, y$ и $z$. В соответствующих выражениях для двумерной модели будет отсутствовать третья переменная: переменная $z$.

2.1. Стационарный процесс. Пусть функция $n(x, y, z)$ описывает плотность ННЗ, находящихся в точке $x, y, z$ после их диффузии в полупроводнике. Она удовлетворяет стационарному дифференциальному уравнению, описывающему диффузию ННЗ в состоянии квазиравновесия:

$$
\Delta n(x, y, z)-\frac{n(x, y, z)}{\lambda^{2}}=-\rho(x, y, z)
$$

и граничным условиям

$$
\begin{gathered}
D \frac{\partial n(x, y, 0)}{\partial z}=v_{s} n(x, y, 0) \\
\lim _{z \rightarrow+\infty} n(x, y, z)=0, \quad \lim _{x \rightarrow \pm \infty} n(x, y, z)=0, \quad \lim _{y \rightarrow \pm \infty} n(x, y, z)=0 .
\end{gathered}
$$

Здесь $\lambda=\sqrt{D \tau}-$ диффузионная длина ННЗ.

Следуя [8], в рассматриваемом случае область генерации ННЗ будем описывать функцией Гаусса $\rho(x, y, z)=G_{0} \tau \phi(x, y, z) / \lambda^{2}$, где $G_{0}$ - частота пульсирующего электронного зонда (и равная ей частота генерации ННЗ), а $\phi(x, y, z)$ - плотность трехмерного нормального распределения Гаусса. В дальнейшем будем считать, что $\rho(x, y, z)=c_{2} \exp \left\{-c_{1}\left(x^{2}+y^{2}+z^{2}\right)\right\}, c_{1}=1 / 2 \sigma^{2}$, $c_{2}=G_{0} \tau / \lambda^{2}(\sigma \sqrt{2 \pi})^{3}$. Пульсирующий электронный пучок используется для повышения отношения «сигнал/шум» при регистрации слабых сигналов люминесценции, возникающей в полупроводниках при их облучении пучком ускоренных электронов или электромагнитным излучением. 
Вопрос о существовании решения задачи (3)-(5) изучался в $[7,8]$. Построением функцию Грина было найдено решение, которое описывается формулой

$$
\begin{aligned}
& n(x, y, z)= \\
& =\int_{-\infty}^{+\infty} \int_{-\infty}^{+\infty} \int_{0}^{+\infty} \rho(\xi, \eta, \zeta)\left(\frac{e^{-\chi R}}{4 \pi R}+\frac{e^{-\chi R_{1}}}{4 \pi R_{1}}-\beta \int_{\zeta}^{+\infty} \frac{\exp \left\{\alpha(\zeta-s)-\chi \sqrt{\rho^{2}+(z+s)^{2}}\right\}}{\sqrt{\rho^{2}+(z+s)^{2}}} d s\right) d \xi d \eta d \zeta,
\end{aligned}
$$

где $R=\sqrt{(x-\xi)^{2}+(y-\eta)^{2}+(z-\zeta)^{2}}, R_{1}=\sqrt{(x-\xi)^{2}+(y-\eta)^{2}+(z+\zeta)^{2}}, \chi=1 / \lambda, \alpha=v_{s} / D$, $\beta=\alpha / 2 \pi, \rho^{2}=(x-\xi)^{2}+(y-\eta)^{2}$.

2.2. Нестационарный прочесс. После того как электронный пучок будет выключен, новые ННЗ генерироваться не будут и будет происходить диффузия имевшихся ННЗ. Этот процесс описывается при помощи следующего дифференциального уравнения в частных производных

$$
\frac{\partial c(x, y, z, t)}{\partial t}=D \Delta c(x, y, z, t)-\frac{c(x, y, z, t)}{\tau}
$$

с начальным условием

$$
c(x, y, z, 0)=n(x, y, z),
$$

где $c(x, y, z, t)$ - концентрация ННЗ в точке с координатами $(x, y, z)$ в момент времени $t, \tau-$ время жизни ННЗ, $\Delta=\partial^{2} / \partial x^{2}+\partial^{2} / \partial y^{2}+\partial^{2} / \partial z^{2}$ - оператор Лапласа, а функция $n(x, y, z)$ определяется выражением (6).

Использованием формулы [8] было получено решение задачи (7), (8):

$$
c(x, y, z, t)=\frac{\exp (-t / \tau)}{(2 \sqrt{D \pi t})^{3}} \int_{-\infty}^{+\infty} \int_{-\infty}^{+\infty} \int_{0}^{+\infty} n(\xi, \eta, \zeta) \exp \left\{-\frac{r^{2}(\xi, \eta, \zeta)}{4 D t}\right\} d \xi d \eta d \zeta
$$

$r(\xi, \eta, \zeta)=\sqrt{(x-\xi)^{2}+(y-\eta)^{2}+(z-\zeta)^{2}}$

Ввиду того, что решение задачи (3)-(5) фактически является составной частью решения задачи (7)-(8), будем рассматривать их как решение одной задачи (3)-(5), (7)-(8).

Для дальнейшего рассмотрения обозначим

$$
\begin{gathered}
\Pi_{x y z}=\{(x, y, z):-\infty<x<+\infty,-\infty<y<+\infty, 0 \leqslant z<+\infty\}, \\
\Pi=\left\{(t, x, y, z): t>0,(x, y, z) \in \Pi_{x y z}\right\} .
\end{gathered}
$$

\section{3. Результаты.}

3.1. Существование и единственность решения задачи диффузии. Следующие теоремы устанавливают единственность задач (3)-(5) и (7)-(8), а, следовательно, и общей задачи (3)-(5), (7)(8).

Теорема 1. Решение задачи (3)-(5) единственно.

Доказательство. Предположим противное. Пусть $n_{1}$ и $n_{2}$ - два различных решения задачи (3)(5). Рассмотрим функцию $u=n_{2}-n_{1}$, которая удовлетворяет следующему дифференциальному уравнению $\Delta u-u / \lambda^{2}=0$ и граничным условиям (4)-(5).

Применив к полученной задаче формулу (6) с $\rho(x, y, z)=0$, получим $u=0$, откуда следует, что $n_{2}=n_{1}$. Полученное противоречие и доказывает единственность решения задачи (3)-(5).

Для двумерной модели доказательство теоремы 1 справедливо без каких-либо изменений. Здесь и далее для двумерной модели в соответствующих формулах координата $z$ отсутствует.

Теорема 2. Решение задачи (7)-(8) единственно. 
Доказательство. Сделав в уравнении (7) и начальном условии (8) замену $c(x, y, z, t)=$ $\exp (-t / \tau) v(x, y, t)$, для функции $v(x, y, z, t)$ получим задачу Коши:

$$
\begin{gathered}
\frac{\partial v}{\partial t}=D \Delta v, \\
v(x, y, z, 0)=n(x, y, z) .
\end{gathered}
$$

Предположим противное. Пусть $v_{1}$ и $v_{2}$ - два различных решения задачи (10)-(11). Рассмотрим функцию $u=v_{2}-v_{1}$, удовлетворяющую дифференциальному уравнению (10) и нулевому начальному условию $u(x, y, z, 0)=0$. Применив к полученной задаче формулу $(9)$, получим $u=0$, откуда $u_{2}=u_{1}$. Противоречие и доказывает единственность решения задачи (7)-(8).

Для двумерной модели доказательство теоремы 2 справедливо без каких-либо изменений.

3.2. Непрерывная зависимость решения от данных задачи. Следуя В. С. Владимирову (см. [2]), непрерывная зависимость решения от данных задачи означает следующее: пусть последовательность данных $D_{k}, k \rightarrow \infty$, и $u_{k}, k=1,2, \ldots$, - соответствующие решения задачи; тогда должно быть $u_{k} \rightarrow u, k \rightarrow \infty$ в смысле надлежащим образом выбранной сходимости. Следующие теоремы устанавливают непрерывную зависимость решения задачи (3)-(5), (7)-(8) от члена в правой части. Доказательство этих теорем основано на единственности задачи, а также на формулах для решений (6), (9). Укажем узловые моменты этих доказательств для трехмерной модели и проведем сравнение с результатами, полученными для двумерной модели.

Теорема 3. Пусть $n_{1}(x, y, z)$ - решение уравнения $\Delta n-n / \lambda^{2}=-\rho_{1}(x, y, z)$ с граничными условиями (4), (5), а $n_{2}(x, y, z)$-решение уравнения $\Delta n-n / \lambda^{2}=-\rho_{2}(x, y, z)$ с граничными условиями (4), (5) и для всех $(x, y, z) \in \Pi_{x y z}$

$$
\left|\rho_{2}(x, y, z)-\rho_{1}(x, y, z)\right| \leqslant \varepsilon .
$$

Тогда для всех $(t, x, y, z) \in \Pi$ справедлива оченка

$$
\left|n_{2}(x, y, z)-n_{1}(x, y, z)\right| \leqslant C \varepsilon, \quad C=\frac{\lambda^{2}+\sqrt{26}}{2} .
$$

Доказательство. Применив формулу (6) для функций $n_{1}$ и $n_{2}$ и учитывая оценку (12), получим

$$
\left|n_{2}(x, y, z)-n_{1}(x, y, z)\right| \leqslant \varepsilon \int_{-\infty}^{+\infty} \int_{-\infty}^{+\infty} \int_{0}^{+\infty}\left|\frac{e^{-\chi R}}{4 \pi R}+\frac{e^{-\chi R_{1}}}{4 \pi R_{1}}-\beta \int_{\zeta}^{+\infty} \frac{e^{\alpha(\zeta-s)-\chi \sqrt{\rho^{2}+(z+s)^{2}}}}{\sqrt{\rho^{2}+(z+s)^{2}}} d s\right| d \xi d \eta d \zeta
$$

Поскольку в (13)

$$
\beta \int_{\zeta}^{+\infty} \frac{e^{\alpha(\zeta-s)-\chi \sqrt{\rho^{2}+(z+s)^{2}}}}{\sqrt{\rho^{2}+(z+s)^{2}}} d s \leqslant \beta \frac{e^{-\chi R_{1}}}{4 \pi R_{1}} \cdot \int_{\zeta}^{+\infty} e^{\alpha(\zeta-s)} d s<\frac{1}{2 \pi} \frac{e^{-\chi R_{1}}}{4 \pi R_{1}},
$$

получаем

$$
\left|n_{2}(x, y, z)-n_{1}(x, y, z)\right| \leqslant \varepsilon \int_{-\infty}^{+\infty} \int_{-\infty}^{+\infty} \int_{0}^{+\infty}\left(\frac{e^{-\chi R}}{4 \pi R}+\frac{3 e^{-\chi R_{1}}}{4 \pi R_{1}}\right) d \xi d \eta d \zeta .
$$

В формуле (14) обозначим

$$
F_{1}(x, y, z)=\int_{-\infty}^{+\infty} \int_{-\infty}^{+\infty} \int_{0}^{+\infty} \frac{e^{-\chi R}}{4 \pi R} d \xi d \eta d \zeta, \quad F_{2}(x, y, z)=\int_{-\infty}^{+\infty} \int_{-\infty}^{+\infty} \int_{0}^{+\infty} \frac{3 e^{-\chi R_{1}}}{4 \pi R_{1}} d \xi d \eta d \zeta
$$

После ряда преобразований для первого из этих интегралов получим

$$
\left|F_{1}\right| \leqslant \frac{1}{2}\left(\frac{1}{\chi^{2}}+\sqrt{26}\right) .
$$


Для $F_{2}$ получим такую же оценку. В результате для неравенства (14) будем иметь

$$
\left|n_{2}(x, y, z)-n_{1}(x, y, z)\right| \leqslant C \cdot \varepsilon, \quad C=\frac{1}{2}\left(\frac{1}{\chi^{2}}+\sqrt{26}\right)=\frac{1}{2}\left(\lambda^{2}+\sqrt{26}\right) .
$$

Теорема 3 доказана.

Для двумерной модели аналогичные соотношения в условиях соответствующей теоремы записываются так (см. [12, 15, 17]):

$$
\left|\rho_{2}(x, y)-\rho_{1}(x, y)\right| \leqslant \varepsilon .
$$

Тогда для всех $(t, x, y) \in \Pi$ справедлива оценка

$$
\left|n_{2}(x, y)-n_{1}(x, y)\right| \leqslant \lambda^{2} \varepsilon .
$$

С точностью до констант формулы (16) и (17) дают одинаковый результат.

Теорема 4. Пусть $c_{1}(x, y, z)$ - решение уравнения (7) с начальным условием $c_{1}(x, y, z, 0)=$ $n_{1}(x, y, z)$, а $c_{2}(x, y, z)$-решение уравнения (7) с начальным условием $c_{2}(x, y, z, 0)=n_{2}(x, y, z)$ и для всех $(x, y, z) \in \Pi_{x y z}$ справедливо неравенство

$$
\left|n_{2}(x, y, z)-n_{1}(x, y, z)\right| \leqslant \delta .
$$

Тогда для всех $(t, x, y, z) \in \Pi$ справедлива оченка

$$
\left|c_{2}(x, y, z, t)-c_{1}(x, y, z, t)\right| \leqslant \delta .
$$

Доказательство. Применив поочередно формулу (9) к задачам для функций $c_{1}$ и $c_{2}$, получим

$$
\begin{aligned}
& c_{1}(x, y, z, t)=\frac{\exp (-t / \tau)}{(2 \sqrt{D \pi t})^{3}} \int_{-\infty}^{+\infty} \int_{-\infty}^{+\infty} \int_{0}^{+\infty} n_{1}(\xi, \eta, \zeta) \exp \left\{-\frac{r^{2}(\xi, \eta, \zeta)}{4 D t}\right\} d \xi d \eta d \zeta \\
& c_{2}(x, y, z, t)=\frac{\exp (-t / \tau)}{(2 \sqrt{D \pi t})^{3}} \int_{-\infty}^{+\infty} \int_{-\infty}^{+\infty} \int_{0}^{+\infty} n_{2}(\xi, \eta, \zeta) \exp \left\{-\frac{r^{2}(\xi, \eta, \zeta)}{4 D t}\right\} d \xi d \eta d \zeta
\end{aligned}
$$

Вычитая второе равенство из первого и учитывая оценку (18), имеем

$$
\begin{aligned}
& \left|c_{2}(x, y, z, t)-c_{1}(x, y, z, t)\right| \leqslant \\
& \leqslant \delta \frac{\exp (-t / \tau)}{(2 \sqrt{D \pi t})^{3}} \int_{-\infty}^{+\infty} \int_{-\infty}^{+\infty} \int_{0}^{+\infty} \exp \left\{-\frac{(x-\xi)^{2}+(y-\eta)^{2}+(z-\zeta)^{2}}{4 D t}\right\} d \xi d \eta d \zeta .
\end{aligned}
$$

Используя теорему Фубини и интеграл Пуассона, оценку (19) перепишем в виде

$$
\left|c_{2}(x, y, z, t)-c_{1}(x, y, z, t)\right| \leqslant \delta \frac{\exp (-t / \tau)}{2 \sqrt{D \pi t}} \int_{0}^{+\infty} \exp \left\{-\frac{(z-\zeta)^{2}}{4 D t}\right\} d \zeta .
$$

В правой части формулы $(20)$ сделаем замену переменной, положив $(\varsigma-z) / 2 \sqrt{D t}=u$. Тогда получим

$$
\left|c_{2}(x, y, z, t)-c_{1}(x, y, z, t)\right| \leqslant \delta \frac{\exp (-t / \tau)}{\sqrt{\pi}} \int_{-z / 2 \sqrt{D t}}^{+\infty} e^{-u^{2}} d u
$$

Поскольку

то из (21) имеем

$$
\int_{-z 2 \sqrt{D t}}^{+\infty} e^{-u^{2}} d u \leqslant \int_{-\infty}^{+\infty} e^{-u^{2}} d u=\sqrt{\pi}
$$

$$
\left|c_{2}(x, y, z, t)-c_{1}(x, y, z, t)\right| \leqslant \delta \exp (-t / \tau)
$$


и, стало быть, при всех $(t, x, y, z) \in \Pi$ справедлива оценка

$$
\left|c_{2}(x, y, z, t)-c_{1}(x, y, z, t)\right| \leqslant \delta .
$$

Теорема 4 доказана.

Для двумерной модели аналогичные соотношения в условиях соответствующей теоремы имеют точно такой же вид $[12,15,17]:$ из соотношения

$$
\left|n_{2}(x, y)-n_{1}(x, y)\right| \leqslant \delta
$$

следует оценка

$$
\left|c_{2}(x, y, t)-c_{1}(x, y, t)\right| \leqslant \delta,
$$

совпадающая с оценкой для трехмерной модели.

Теорема 5. Пусть $c_{1}(x, y, z)$ - решение уравнения (7) с начальным условием $c_{1}(x, y, z, 0)=$ $n_{1}(x, y, z)$, a $c_{2}(x, y, z)$-решение уравнения (7) с начальным условием $c_{2}(x, y, z, 0)=n_{2}(x, y, z)$ и для всех $(x, y, z) \in \Pi_{\text {хуz }}$ функции $n_{1}(x, y, z)$ и $n_{2}(x, y, z)$ удовлетворяют условиям теоремы 3. Тогда для всех $(t, x, y, z) \in \Pi$ справедлива оченка

$$
\left|c_{2}(x, y, z, t)-c_{1}(x, y, z, t)\right| \leqslant C \cdot \varepsilon, \quad C=\frac{1}{2}\left(\lambda^{2}+\sqrt{26}\right) .
$$

Доказательство. Доказательство теоремы 5 сразу же следует из теорем 3 и 4.

Для двумерной модели аналогичные соотношения в условиях соответствующей теоремы при выполнении условий теоремы 3 для всех $(t, x, y) \in \Pi$ записываются так (см. $[12,15,17])$ :

$$
\left|c_{2}(x, y, t)-c_{1}(x, y, t)\right| \leqslant \lambda^{2} \cdot \varepsilon .
$$

С точностью до констант формулы (23) и (24) дают одинаковый результат.

4. Заключение. Проведено сравнительное исследование качественных свойств двумерной и трехмерной математических моделей диффузии частиц (неравновесных неосновных носителей заряда, экситонов), возбуждаемых пульсирующим остро сфокусированным электронным зондом в однородном полупроводниковом материале. С использованием методов качественной теории дифференциальных уравнений для математической модели изучаемого физического явления проведено исследование ее корректности, доказана непрерывная зависимость решения (выходных данных) от входных данных, получены оценки, позволяющие оценить влияние погрешностей в исходных данных на распределение диффундирующей примеси. Полученные результаты могут быть использованы при планировании эксперимента в электроннозондовых технологиях.

\section{СПИСОК ЛИТЕРАТУРЫ}

1. Бонч-Бруевич В. Л., Калашников С. Г. Физика полупроводников. - М.: Наука, 1990.

2. Владимиров В. С., Жаринов В. В. Уравнения математической физики. - М.: Физматлит, 2004.

3. Михеев Н. Н., Никоноров И. М., Петров В. И., Степович М. А. Определение электрофизических параметров полупроводников в растровом электронном микроскопе методами наведенного тока и катодолюминесценции // Изв. АН СССР. Сер. физ. - 1990. - 54, № 2. - С. 274-280.

4. Михеев Н. Н., Петров В. И., Степович М. А. Количественный анализ материалов полупроводниковой оптоэлектроники методами растровой электронной микроскопии// Изв. РАН. Сер. физ. $-1991 .-55$, № $8 .-$ C. $1474-1482$.

5. Михеев H. H., Степович M. А. Распределение энергетических потерь при взаимодействии электронного зонда с веществом// Заводская лаборатория. Диагностика материалов. $-1996 .-62$, № 4 . C. $20-25$.

6. Панков Ж. Оптические процессы в полупроводниках. - М.: Мир, 1973.

7. Поляков А. Н., Степович М. А., Туртин Д. В. Математическое моделирование катодолюминесценции экситонов, генерированных узким электронным пучком в полупроводниковом материале// Изв. РАН. Сер. физ. - 2016. - 80, № 12. - С. 1629-1633.

8. Поляков А. Н., Степович М. А., Туртин Д. В. Трехмерная диффузия экситонов, генерированных электронным пучком в полупроводниковом материале: результаты математического моделирования// Поверхность. Рентген. синхротрон. нейтрон. исслед. - 2015. - 12. - С. 48-52. 
9. Поляков A. H., Noltemeyer M., Hempel T., Christen J., Cmenович M. А. Двумерная диффузия и катодолюминесценция экситонов, генерированных электронным пучком в полупроводниковом материале: результаты математического моделирования / / Поверхность. Рентген. синхротрон. нейтрон. исслед. 2012. - 11. - C. 35-40.

10. Поляков A. H., Noltemeyer M., Hempel T., Christen J., Cmenович M. A. Катодолюминесцентные экспериментальные исследования транспорта экситонов в нитриде галлия// Изв. РАН. Сер. физ. - 2012. - 76, № 9. - C. 1082-1085.

11. Поляков A. H., Noltemeyer M., Hempel T., Christen J., Cmenович M. A. Оценка значений электрофизических параметров полупроводниковых материалов по результатам измерений катодолюминесценции экситонов // Прикл. физ. - 2012. - 6. - С. 41-46.

12. Степович М. А., Туртин Д. В., Серегина Е. В., Поляков А. Н. О качественных характеристиках двумерной математической модели диффузии неосновных носителей заряда, генерированных низкоэнергетическим электронным зондом в однородном полупроводниковом материале// Сб. тр. междунар. науч. конф. «Актуальные проблемы прикладной математики, информатики и механики» (17-19 декабря 2018 г., Воронеж). - Воронеж: Воронеж. гос. ун-т, 2019. - С. 127-133.

13. Туртин Д. В. Теоремы единственности решения задачи Коши для эволюционных уравнений и систем с растущими коэффициентами/ Дисс. на соиск. уч. степ. канд. физ.-мат. наук - Владимир: ВГУ, 2012.

14. Noltemeyer M., Bertram F., Hempel T., Bastek B., Polyakov A. N., Christen J., Brandt M., Lorenz M., Grundmann M. Excitonic transport in ZnO// J. Mater. Res. - 2012. - 27, № 17. — P. 2225-2231.

15. Polyakov A. N., Smirnova A. N., Stepovich M. A., Turtin D. V. Qualitative properties of a mathematical model of the diffusion of excitons generated by electron probe in a homogeneous semiconductor material// Lobachevskii J. Math. - 2018. — 39, № 2. — P. 259-262.

16. Stepovich M. A., Amrastanov A. N., Seregina E. V. and Filippov M. N. Mathematical modelling of heating of homogeneous metal targets with a focused electron beam// J. Phys. Conf. Ser. — 2019. — 1163. — P. 012014-1-012014-6.

17. Stepovich M. A., Turtin D. V., Seregina E. V. and Polyakov A. N. On the qualitative characteristics of a two-dimensional mathematical model of diffusion of minority charge carriers generated by a lowenergy electron beam in a homogeneous semiconductor material// J. Phys. Conf. Ser. — 2019. — 1203. — P. 012095-1-012095-8.

Степович Михаил Адольфович

Калужский государственный университет им. К. Э. Циолковского

E-mail: m.stepovich@rambler.ru

Туртин Дмитрий Витальевич

Российский экономический университет им. Г. В. Плеханова, Ивановский филиал

E-mail: turtin@mail.ru

Серегина Елена Владимировна

Московский государственный технический университет им. Н. Э. Баумана

(национальный исследовательский университет), Калужский филиал

E-mail: evfs@yandex.ru 